\title{
END OF LIFE
}

\section{Scale of levels of care versus DNR orders Debate D Vanpee, C Swine}

1 $\mathrm{n}$ his paper, which we read with interest, Cherniack argues that there is a worldwide increase in the use of do not resuscitate (DNR) orders in the care of the elderly. ${ }^{1}$ As geriatricians in an emergency department and a geriatric department we are concerned by this important ethical topic, and we understand that this increase is a reflection of the demographic increase in frail very old persons. The elderly must be offered the best care, and age as such should not be used as a decision criterion in order to exclude them from the best care. Nevertheless, care which is believed to be harmful and treatments which are considered futile must not be offered, and in some circumstances must not even be discussed. In the setting of the care of the frail elderly, we believe the use of simple DNR orders to be inappropriate and restrictive, because they fail to outline the kind of care and the extent of the care that the patient actually needs. We prefer a more nuanced approach based on a scale describing positively the contents of care at four different levels: terminal, palliative, usual, and intensive, only the last to include cardiopulmonary resuscitation (CPR). Perhaps a more positive formulation of such orders, as presented here, may contribute to changing attitudes and thus reduce the risk of "abandonment of the patient", which is potentially linked to the increasing use of simple DNR orders.

Accordingly, we want to make several additional comments and one suggestion about the use of DNR orders in the care of the elderly.

1) The older adult population is heterogeneous, and rational attitudes towards the fit elderly are not necessarily appropriate towards some frail very old persons. The numbers of patients entering our hospital, who constitute the most vulnerable elderly population, that is those over 75 , is increasing rapidly. The proportion of these patients admitted to the A\&E department increased from $12 \%$ in 1996 to $18 \%$ in $2002 .^{2}$ Several have severe comorbidities, functional dependency, and are very frail. This increasing proportion of frail elderly patients being admitted to hospital could perhaps explain, at least in part, the increasing use of DNR orders in the elderly worldwide, as reported by Cherniack in his paper.

2) During hospitalisation, there is a need for early discussions about the level of care needed by these frail patients. All competent elderly patients have the right to accept or refuse cardiopulmonary resuscitation after they have been informed of the benefits and risks. This does not mean that as doctors we need to discuss all decisions with the patient, particularly when the discussion is likely to be harmful or when CPR is likely to be futile. Patients expect their doctors to make the best decision for them, and it is generally accepted that if the treatment offers little value, it does not need to be offered to the patient; there is no obligation to provide treatments believed to be futile. ${ }^{3}$ Age alone is not a contraindication for therapeutic interventions, but only one among several factors to be considered in determining the risks and benefits of a given treatment. Outcomes of CPR have been shown to be very poor in frail elderly patients. Many studies have demonstrated that age alone is not the main determinant of CPR success. More important are the patient's prearrest comorbidity, the specific initial dysrhythmia, and the underlying cardiac disorder. Elderly patients who are functionally independent without chronic illnesses have a better prognosis after CPR than frail elderly nursing home residents. Patients with ventricular fibrillation as the initial dysrhythmia have the best chance of survival with CPR compared to patients with asystole and patients with electrical mechanical dissociation. Patients with acute myocardial infarction are more likely to benefit from CPR than elderly patients with pneumonia.

3) Data in the literature have consistently highlighted major deficiencies, particularly the risk of "abandonment of the patient", where DNR orders are given in order to limit the use of cardiopulmonary resuscitation. Lipton showed in his study that although many DNR policies consider DNR status fully compatible with aggressive care, in current clinical practice the DNR order usually leads to less intensive care. ${ }^{4}$ Therefore, because DNR decisions carry this risk of influencing other treatment decisions that are potentially useful for the patient, we do not use such orders on our geriatric ward. Indeed, the DNR order implies "do not" whereas we implement positive actions for these patients. Instead of the negative approach, we prefer to use a scale which outlines levels of care describing positively what must be done..$^{5}$ Thus, a systematic, clinically driven, planning process is implemented that involves four steps for our frail elderly patients: terminal phase level; palliative care level; usual care level, and intensive care level (see the Framework for a four level continuum of care below). Our approach is defined through team discussions, which also take into account patients' and relatives' values. For each phase we have specified positive therapeutic actions: the care provided in the terminal phase
D Vanpee, C Swine, Emergency Department and Geriatric Department, respectively Université Catholique de Louvain, Yvoir, Belgium

Correspondence to: Prof D Vanpee, Emergency Department, UCL Mont-Godinne, 5530 Yvoir, Belgium; Dominique.vanpee@ rean.ucl.ac.be

Accepted for publication 18 March 2003 


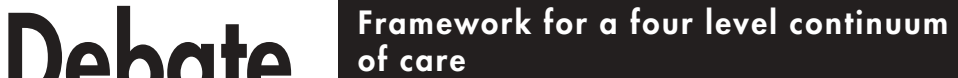

Suggested framework for an interdisciplinary and therapeutic plan which requires additional adjustments for each individual case, and regular reassessment of each case.

Terminal level content of care:

Body hygiene, pain control, body position, mouth care, management of emotions.

Palliative level content of care-above mentioned content, in addition:

Skin integrity care; mobilisation, transfers, and exercises; elimination control; hydration and nutrition by mouth; symptoms control (fever, nausea, dyspnoea); medical or surgical interventions required for symptom control.

Usual level content of care-above mentioned content, in addition:

Usually required diagnostic procedures; all required medical treatments with intravenous route if necessary; tube or parenteral artificial nutrition if required; surgical or technical procedures required to improve functional capacity.

Intensive level content of care-above mentioned content, in addition:

Advanced required diagnostic and therapeutic procedures; full cardiopulmonary resuscitation with sustained manoeuvres when required. remains the basis of all care at higher levels. This careful planning of a continuum of care tends to ensure that older patients receive the most appropriate level of care at the most appropriate time and place.

\section{REFERENCES}

1 Cherniack EP. Increasing use of DNR orders in the elderly worldwide: whose choice is it? J Med Ethics 2002;28:303-7.

2 Vanpee D, Swine C, Vandenbossche P, et al. Epidemiological profile of geriatric patients admitted to the emergency department of a university hospital localised in a rural area. Eur J Emerg Med 2001;8:301-4.

3 Waisel DB, Truog RD. The cardiopulmonary resuscitation not indicated order: futility revisited. Ann Intern Med 1995; 122:304-8.

4 Lipton HL. Do not resuscitate decisions in a community hospital. Incidence, implications, and outcomes. JAMA 1986;256:1164-9.

5 Voyer G. Ethique clinique. In: Arcand-Hébert, ed. Précis pratique de gériatrie [2nd ed]. Edisem: Maloine, $A Q$, 1997:675-87.

\section{$\mathrm{ECHO}$}

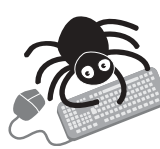

Please visit the Journal of Medical Ethics website [www. imedethics. com] for a link to the full text of this article.

\section{Ethical points from the SARS outbreak in Toronto}

n response to the 2003 outbreak of severe acute respiratory syndrome (SARS) in Toronto, Canada a working party of bioethicists and other professionals was set up to identify the main ethical issues raised by the outbreak. They reached a consensus on ten key ethical values involved: individual liberty, protection of the public, proportionality, reciprocity, transparency, privacy, protection of communities from stigmatisation, duty to provide care, equity, and solidarity. The applicability of these values is explored in relation to four practical professional issues and one global consideration (air travel):

- A hospital worker is quarantined at home for 10 days but fears for her job and her home

Individual liberty must be balanced against protection of the public. Proportionality demands that this be done fairly and reasonably. Transparency requires that people involved be fully informed and reciprocity means that people must not suffer to an unfair extent economically as a result of complying with public health measures.

- A nurse thinks she might have SARS but travels to work on a commuter train.

Should she be named?

Proportionality demands that the nurse's privacy be respected unless breaching that privacy would serve an important purpose for the community. The naming of the Chinese woman who carried SARS to Canada exposed the Chinese community to stigmatisation.

- A health worker is afraid of contracting SARS and transmitting it to her family

Is the duty of health workers to provide care unlimited? The working party was unable to resolve this issue. Reciprocity requires that they be given appropriate support and protection. An insurance fund to cover health workers during epidemics might be considered.

- Other patients had important operations cancelled during the outbreak

Collateral damage during an outbreak is unavoidable. There must be as much equity as possible between the demands of the outbreak and the interests of other patients.

- The disease was spread by air travel

Global cooperation (solidarity) is necessary to deal with serious infectious diseases. Countries should be transparent about diseases that might spread to other countries.

Several of the issues raised do not have readily available answers. The authors of this paper call for further research, both conceptual and empirical. They have constructed a decision tool based on the ten key ethical values.

\ BMJ 2003;327:1342-1344. 